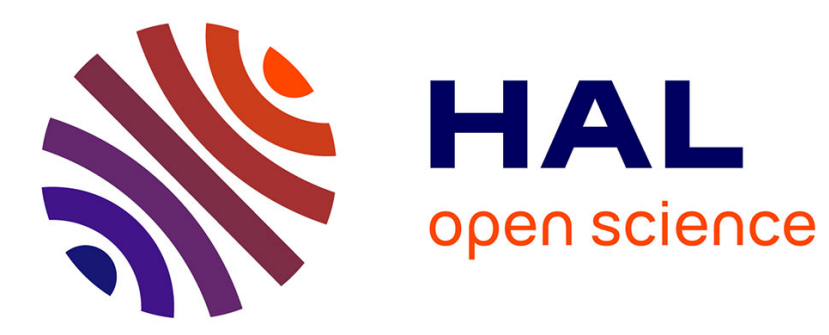

\title{
Repenser l'article 714 du Code civil français comme une porte d'entrée vers les communs
}

Marie-Pierre Camproux Duffrene

\section{To cite this version:}

Marie-Pierre Camproux Duffrene. Repenser l'article $714 \mathrm{du}$ Code civil français comme une porte d'entrée vers les communs. 2018. halshs-01933631

\section{HAL Id: halshs-01933631 \\ https://shs.hal.science/halshs-01933631}

Preprint submitted on 23 Nov 2018

HAL is a multi-disciplinary open access archive for the deposit and dissemination of scientific research documents, whether they are published or not. The documents may come from teaching and research institutions in France or abroad, or from public or private research centers.
L'archive ouverte pluridisciplinaire HAL, est destinée au dépôt et à la diffusion de documents scientifiques de niveau recherche, publiés ou non, émanant des établissements d'enseignement et de recherche français ou étrangers, des laboratoires publics ou privés. 
Marie-Pierre Camproux Dufffrène, «Repenser l'article 714 du Code civil ${ }^{1}$ comme une porte d'entrée vers les communs » In Dossier "Les communs - Actualité et vitalité de la notion à la croisée des enjeux environnementculture", dirigé par Delphine Misonne, Revue Internationale d'Etudes Juridiques, Bruxelles, à paraître

\section{Repenser l'article 714 du Code civil français comme une porte d'entrée vers les communs}

Résumé : En droit français, de la chose commune au commun, il peut sembler n'y avoir qu'un pas. Mais les catégories du droit civil sont-elles suffisamment perméables aux enjeux sociétaux et écologiques (I) ? L'article 714 CC offre un potentiel innovant (II), conciliable avec une théorie des communs (III), telle que proposée ici par l'auteure sur un mode résolument utopique, à condition d'en provoquer l'adaptation et la malléabilité.

«Les évolutions techniques, écologiques, urbaines rendent nécessaire une construction véritablement actuelle des modes d'organisation et de distribution des droits. Le fait que de nombreuses ressources soient devenues rares, que certains espaces soient plus fragiles, rend d'autant plus nécessaire la mise en commun de leur usage et de leur administration, puisque notre vulnérabilité et celle des choses sont indissociables. ${ }^{1}$

Dans ce dossier sur les communs, nos propos seront focalisés sur l'environnement naturel dans sa diversité biologique actuelle. L'analyse portera sur la possible application de l'article $714 \mathrm{du}$ Code civil français à la biodiversité. Cet article 714 du Code civil français créé par la loi du 19 avril 1803, promulguée le 29 avril 1803, édicte :

"Il est des choses qui n'appartiennent à personne et dont l'usage est commun à tous.

Des lois de police règlent la manière d'en jouir. »

Il faut préciser également que repenser l'article 714 du Code civil n'est pas le réécrire mais effectuer en 2018 une relecture d'un texte de 1803 inséré dans le Code civil en 1804. À l'heure du changement climatique, de la perte de biodiversité ${ }^{2}$, de l'anthropocène ${ }^{3}$, de l'invention de l'absence de perte nette de biodiversité et des premiers pas vers la marchandisation de biodiversité via l'offre de compensation et les sites afférents ${ }^{4}$. A l'heure d'une modification de

\footnotetext{
${ }^{1}$ Pierre Charbonnier et Daniela Festa, « Biens communs, beni comuni », Tracés. Revue de Sciences humaines [En ligne], \#16|2016, mis en ligne le 01 janvier 2017, consulté le 26 février 2018. URL: http://journals.openedition.org/traces/6622

2 Anne-Sophie Boutaud, «Biodiversité : l'état d'urgence », CNRS Le Journal, 29 mars 2018, https://lejournal.cnrs.fr/print/1826

${ }^{3}$ Notamment (dir. R. Beau et C. Larrère), Penser l'anthropocène, éd. SciencesPo, Paris, Les presses, 2018

${ }^{4}$ La loi du 8/8/2016 pour la reconquête de la biodiversité, de la nature et des paysages a entériné la possibilité de recourir à l'acquisition d'unités de compensation en vue de satisfaire à une obligation de compensation. Créées dans le cadre d'un site de compensation, ces unités représentent des opérations de restauration ou de développement d'éléments de biodiversité mises en place dans le but d'être vendues à des maitres d'ouvrage. Cette option, bien que présentant un certain nombre d'avantages de par la mutualisation et l'anticipation des besoins compensatoires qu'elle vise, n'est pas sans poser des risques d'échec et de manipulation, eu égard au caractère relativement peu fongible de la biodiversité et à la difficulté de concilier les intérêts publics de conservation de la nature avec une logique marchande
} 
l'article L110-1 du code français de l'environnement opérée par la loi du 8/8/2016 pour la reconquête de la biodiversité, de la nature et des paysages - loi qui a non seulement défini les termes de biodiversité et introduit la notion d'êtres vivants comme partie du patrimoine commun de la nation regroupant ainsi vivant humain et non humain dans l'article L 110-1 I mais également inséré dans 1'art L110-1 II les principes de solidarités écologiques et de l'utilité durable ${ }^{5}$. Et pour finir à l'heure d'une montée en puissance de ce que l'on appelle les communs envisagés comme une troisième voie entre le marché et l'État selon Elinor Ostrom ${ }^{6}$. En effet, les différents éléments environnementaux se faisant rares ou étant dégradés, la dépendance humaine s'accroit. Les conflits d'usage se développent et se font de plus en plus violents, leur appropriation entraîne des tensions dans les relations entre les usagers et plus leur état se dégrade plus la pression augmente ${ }^{7}$.

Dans ce contexte d'urgence écologique ${ }^{8}$, repenser l'article 714 du Code civil repose sur une double réflexion. D'une part le droit est un construit social ${ }^{9}$, il n'est pas immuable et doit

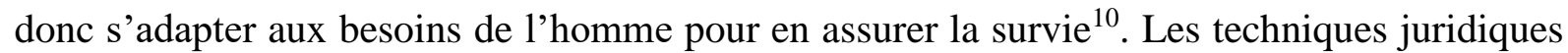
répondent à des finalités, ou bien sont mises au service de celles-ci ${ }^{11}$. Et aujourd'hui plus qu'hier et moins que demain, la protection de l'environnement, et de manière concomitante la survie de l'espèce humaine, sont des priorités absolues. D'autre part, si la planète peut assurément survivre à l'espèce humaine, l'inverse n'est pas vrai, l'homme étant en communauté de destin avec le reste du monde vivant. Il faut donc repenser notre vision d'un monde désormais fini. Réfléchir sur l'article 714 du Code civil en fait partie.

Cet article 714 est, depuis 1804, le fief ou l'article refuge des choses communes dans le Code civil.

En 2009, nous avions essayé de démontrer que s'il est classique d'accorder la qualification de chose commune à l'eau courante ou à l'air, il fallait y adjoindre la biodiversité ${ }^{12}$ au regard du contexte de crise écologique qui n'existait pas en 1803. En effet, la pollution de l'air, des eaux, la stérilisation des sols, la disparition des espèces changent la donne. Cette catégorie de chose commune du Code civil peut aujourd'hui être moteur dans une réflexion globale. Elle peut accompagner la recherche de nouveaux équilibres civilisationnels entre

\footnotetext{
${ }^{5}$ Article L110-1 II $6^{\circ}$ du Code de l'environnement «Le principe de solidarité écologique, qui appelle à prendre en compte, dans toute prise de décision publique ayant une incidence notable sur l'environnement des territoires concernés, les interactions des écosystèmes, des êtres vivants et des milieux naturels ou aménagés ;

$7^{\circ}$ Le principe de l'utilisation durable, selon lequel la pratique des usages peut être un instrument qui contribue à la biodiversité. »

${ }^{6}$ Une troisième voie entre l'Etat et le marché, Echanges avec Elinor Ostrom, Col. Nature et société, éd. Quae, 2017, 151 pages, https://creativecommons.org/licenses/by-nc-nd/2.0/fr/

${ }^{7}$ S. Gutwirth et I. Stengers, «Le droit à l'épreuve de la résurgence des commons », RJE 1/2016, p.327

${ }^{8}$ V. les 5 rapports d'évaluation scientifique et politique établis pour la Sixième session Medellin (Colombie) de la Plateforme Intergouvernementale scientifique et politique sur la biodiversité et les services écosystémiques 1824 mars 2018

${ }^{9}$ Intervention de Marie Cornu «Exposer la pluralité de sens : un dictionnaire des communs », séminaire organisé par Delphine Misonne sur «Actualités des communs en droit de l'environnement et de la culture, 28 novembre 2017, CEDRE, Université Saint Louis, Bruxelles.

${ }^{10}$ Serge Gutwirth et Isabelle Stengers, «le droit à l'épreuve de la résurgence des commons, RJE 1/2016, p. 306 à 343.

${ }^{11}$ F. Ost, « A quoi sert le droit ?; Usages, fonctions finalités », Bruxelles, Bruylant, 2016

${ }^{12}$ M.-P. Camproux Duffrène, « La protection de la biodiversité via le statut de res communis », Revue Lamy Droit civil, janv. 2009, Perspectives, p. 68 à 74
} 
humain et non humain, vivant et non vivant, mais également de nouveaux équilibres juridiques entre propriété et communalité ${ }^{13}$, entre droit individuel et droit trans-individuel ${ }^{14}$.

En matière environnementale, une lecture actualisée de l'article 714 du Code civil (I) devrait promouvoir une activation de sa mise en œuvre (II). Dans la mesure où la chose commune peut être perçue comme l'objet d'un « commun universel » auquel peut être appliqué un régime juridique primaire, elle peut servir la réflexion sur les communs (III).

\section{I-. Une lecture actualisée de l'article 714 du Code civil institutionnalisant la chose commune}

Cette relecture permet non seulement de caractériser la chose commune (A) mais également de mettre en exergue les lignes directrices du régime juridique y afférent (B).

\section{A. La caractérisation de la chose commune du fait des liens en jeu}

La qualification de chose commune en matière environnementale s'applique classiquement à l'air, l'eau mais peut également l'être à des éléments incorporels tels que la biodiversité, et l'écosystème planétaire ${ }^{15}$. Cependant selon $\mathrm{F}$. Ost le monde des communs est un monde relationnel, l'accent n'est pas mis sur le sujet ou sur l'objet de droit mais sur la relation ${ }^{16}$. Dans cette mesure, il faut relever que dans le domaine de l'environnement naturel, les relations chose-environnement et hommes sont particulières tant sur le plan factuel (1) que sur le plan juridique (2).

\section{Un lien factuel}

Que l'environnement naturel appartienne à cette catégorie de chose commune semble aller de soi, tout d'abord matériellement mais également en raison de son caractère vital pour l'homme, « indispensable à la vie » selon Sériaux ${ }^{17}$. Il faut noter que l'article 715 , bien que de manière implicite, ne laisse pas place au doute quant à cette intégration du vivant dans cette

\footnotetext{
${ }^{13}$ V. (dir. Marie Cornu, Fabienne Orsi et Judith Rochfeld), Le dictionnaire sur les biens communs, Paris, PUF 2017

${ }^{14}$ M-P Camproux Duffrène «la représentation de l'intérêt collectif environnemental devant le juge civil : après l'affaire Erika et avant l'introduction dans le Code civil du dommage causé à l'environnement, in La représentation de la nature devant le juge ; approches comparatives et prospectives, VertigO - la revue électronique en sciences de l'environnement [En ligne], Hors-série 22 | septembre 2015, mis en ligne le 10 septembre 2015, consulté le 28 février 2018. URL : http://journals.openedition.org/vertigo/16320

${ }^{15}$ MP Camproux Duffrène, «La protection de la biodiversité via le statut de res communis », Revue Lamy Droit civil, janv. 2009, Perspectives, p. 68 à 74

${ }^{16}$ Intervention de F. Ost, « Du commun à la personnalité juridique accordée à la nature » in séminaire organisé par Delphine Misonne sur « Actualités des communs en droit de l'environnement et de la culture, 28 novembre 2017, CEDRE, Université Saint Louis, Bruxelles.

${ }^{17}$ A. Sériaux, «La notion de choses communes, nouvelles considérations juridiques sur le verbe avoir », in J.-Y. Chérot et al., Droit et environnement. Propos pluridisciplinaires sur un droit en construction, Aix-Marseille, éd. PUAM 1995, p. 35
} 
catégorie de chose commune en précisant que «La faculté de chasser ou de pêcher est également réglée par des lois particulières ».

Ce lien entre la chose commune, environnement naturel et homme est fait d'interdépendance et de solidarité contrairement à l'idée que l'homme peut maîtriser la nature et l'assujettir. L'environnement naturel (écosystème planétaire, biodiversité) et l'homme sont en effet en communauté de destin ${ }^{18}$, leurs sorts sont liés. Il faut préciser que du côté de l'homme, ce lien factuel est divers, il n'est pas seulement spatial ou géographique ou encore purement biologique, il est neurologique ${ }^{19}$, spirituel, culturel, cultuel $^{20}, \ldots$ Il sous-tend la condition humaine.

Dans notre société de consommation et en urbanisation constante déconnectée de notre naturalité au regard de la finitude de notre planète, et de l'état de dégradation des écosystèmes mettant en péril leur capacité de résilience, cette relation d'interdépendance et de solidarité est mise à mal et l'irréversibilité de certaines atteintes à la chose commune pose l'urgence de rétablir l'équilibre de cette relation. Or si cette chose commune est vitale pour l'homme, son fonctionnement permettant la survie de l'espèce humaine dépend aujourd'hui essentiellement de lui et de sa capacité à le protéger de ses propres activités.

De plus, cette relation homme-environnement naturel est complexe dans la mesure où se superpose au lien que chaque homme entretient avec la chose commune ceux des autres hommes avec cette même chose commune. Cette superposition, mise en image par ces schémas, oblige alors les hommes entre eux du fait de leur relation avec la chose commune.

\footnotetext{
${ }^{18}$ R. Mathevet, La solidarité écologique, ce lien qui nous oblige, Arles, Actes Sud 2012, p. 88

${ }^{19} \mathrm{https}$ //theconversation.com/si-vous-etes-terrien-vous-avez-besoin-de-la-nature-85728

${ }^{20} \mathrm{R}$. Lafargue, « Le préjudice civilisationnel pour atteinte à l'environnement. Droit au cadre naturel et réalités socioculturelles : interdépendances et interdisciplinarité », Droit et société, 2010/1 n 74, p. 151-169.
} 


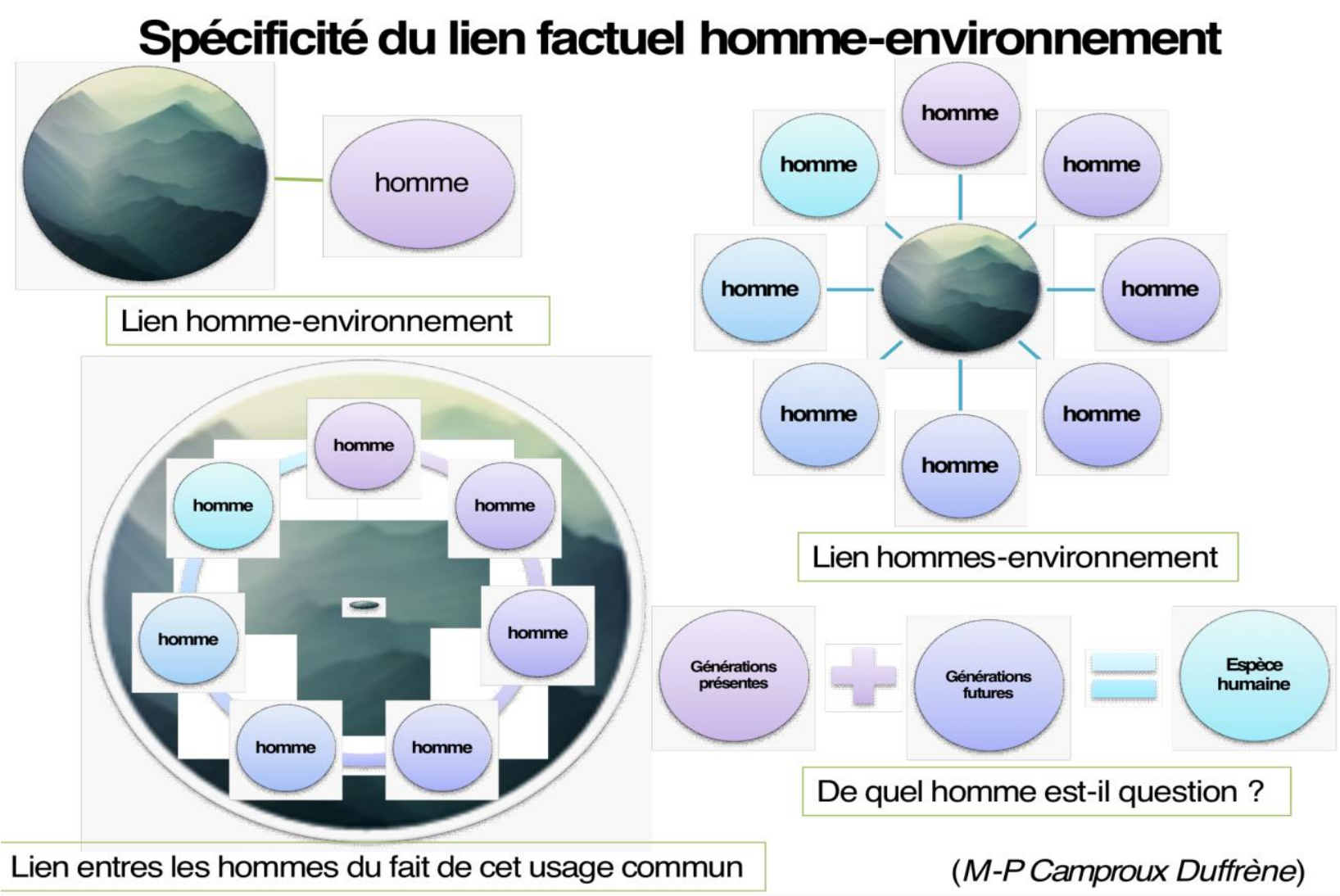

$\mathrm{Au}$ regard de ces relations superposées et de la nature vitale du maintien du fonctionnement de la chose commune, les atteintes irréversibles à la chose commune sont dès lors gravissimes pour la chose commune en premier lieu, et consécutivement pour l'humanité entière composée des générations présentes, mais également des générations futures étant donné leurs dépendances.

Comment le droit traduit-il juridiquement ces liens via l'article 714 du Code civil ?

\section{Une relation juridique au regard de l'article 714}

Tout d'abord, la place de cet article traitant de la chose commune dans le Code civil est importante ${ }^{21}$. Il se situe hors du livre 2 relatif « aux biens et des différentes modifications de la propriété », mais dans le préambule du livre 3 du Code civil intitulé « Des différents manières dont on acquiert la propriété », parmi les dispositions qui présentent les éléments qui ne sont pas, plus ou pas encore appropriés donc hors de la propriété (avec les choses non encore appropriés : les choses sans maître ou les trésors).

Ensuite, l'article 714 précise ce qu'est une chose commune. D'une part, c'est une chose « qui n'appartient à personne », donc sans propriétaire, ce qui l'exclut de la catégorie des biens.

\footnotetext{
${ }^{21}$ Selon J. Rochfeld, entrée « chose commune (approche juridique) », in (dir. Marie Cornu, Fabienne Orsi et Judith Rochfeld), Le dictionnaire sur les biens communs, Paris, PUF 2017, p. 177
} 
Elle est logiquement hors patrimoine ${ }^{22}$ au sens civiliste du terme puisque sans valeur monétaire. Cette chose commune échappe en tant qu'entité à l'échange, à la marchandisation et la monopolisation ${ }^{23}$. D'autre part, l'article précise, ce qui n'a pas toujours été relevé, que cette chose, qui ne fait pas l'objet de droit de propriété, est néanmoins objet d'usage (« dont l'usage est commun à tous »). Ainsi, la chose commune n'est ni bien ni personne juridique mais une chose, objet d'usage par chaque homme et l'ensemble de l'espèce humaine. Cet usage commun correspond alors à des usages, comme nous l'avons dit précédemment, multiples et variés au regard de la dépendance de l'homme vis-à-vis de l'environnement.

Il est ajouté au second alinéa de l'article 714 du Code civil que les lois de police règlent la manière d'en jouir. Cet alinéa est instructif. Il précise que cet usage correspond bien à la fois à l'usus et au fructus qui est le droit de bénéficier des fruits de la chose. Il précise aussi qu'il faut rechercher dans les lois de police comment cette jouissance est encadrée. Cette référence à ces lois de polices est peu explicite en droit civil français. Au regard de l'article 714 du Code civil belge, il s'agirait de lois qui ne sont pas forcément des lois pénales ni de loi de police administratives, en revanche ce devrait être des lois impératives qui s'imposent à tous sans forcément l'adhésion de chacun, qui permettent l'action et qui peuvent évoluer au regard de la caractérisation de la chose commune ${ }^{24}$. Elles peuvent alors se trouver, en ce qui concerne la chose commune «environnement», en droit français notamment dans le Code de l'environnement comme dans le Code civil. Cela étant dit, l'alinéa premier de l'article 714 nous livre les lignes directrices de ce régime juridique qui peut être mis en œuvre par ces lois de polices.

\section{B. Les lignes directrices du régime juridique applicable à la chose commune}

La chose commune n'est pas la propriété de tous comme cela a pu être largement répandu, ni l'objet d'une propriété collective. Au contraire, à la lecture attentive de l'article 714 l'homme ne peut pas être propriétaire ou copropriétaire de la chose, il n'en a qu'un usage commun avec tous (1). Et ce droit d'usage commun a comme contrepartie une obligation, celle de conserver la chose pour maintenir cet usage commun (2).

\section{Un usage spécifique de la chose commune}

L'usage dont il est question dans l'article 714 du Code civil n'apporte à l'homme que des prérogatives réduites sur la chose, prérogatives qu'il partage de surcroît avec tous.

\footnotetext{
${ }^{22}$ Le patrimoine selon Aubry et Rau regroupant l'ensemble des biens et des obligations ayant une valeur monétaire d'une même personne juridique.

${ }^{23}$ Il faut bien distinguer la chose commune de certaines de ses parties ou composantes détachables ou de ses productions, V. troisième partie

${ }^{24}$ Benoit Jadot, «L'article 714 du Code civil et la protection de l'environnement », in F. Ost et S. Gutwirth, Quel avenir pour le droit de l'environnement ?, Bruxelles, Publ. Faculté universitaires Saint Louis 1996, p. 68
} 
Au regard de l'article 714 du Code civil, l'homme est un simple usager de la chose commune. Un usager dispose d'un pouvoir limité sur la chose à la différence du propriétaire qui a un droit exclusif (personnel) et absolu (complet : usus, fructus, abusus) fondé sur l'article 544 du Code civil. Le titulaire du droit d'usage n'a pas l'abusus, c'est-à-dire le droit de disposer et d'aliéner la chose, de la dégrader ou de la polluer.

En tant qu'usager, il a en revanche un droit de jouir de la chose pour ses besoins vitaux dans une limite raisonnable. Il est possible d'emprunter au Code civil certaines règles applicables au droit d'usage classique, notamment celle de respecter une obligation de jouir raisonnablement de la chose $\mathrm{c}^{25}$.

Il faut noter qu'en tant qu'usager, son droit de prélèvement est limité aux fruits (renouvelables et sans répercussion sur le bon fonctionnement de la chose) qui deviennent la propriété de l'usager à l'exclusion des produits (qui entament ou dont le prélèvement porte atteinte à la substance de la chose dont il ne dispose pas).

Chaque homme doit pouvoir en tirer les utilités nécessaires et donc bénéficier d'un droit à la non-exclusion. Ce qui révèle d'ores et déjà que cet usage ne peut être réalisé sans contextualisation sociale et que ce lien juridique correspond à cette superposition de liens factuels précédemment schématisés. En effet, cet usage se révèle juridiquement complexe dans la mesure où il est commun à tous, ce qui influe juridiquement sur ses effets.

En effet, l'article 714 précise que cet usage est « commun à tous ».

Cet usage doit permettre un accès de tous en fonction des besoins de chacun mais également de ceux des autres. Cet usage doit prendre en compte l'autre, il peut être limité par celui des autres. Ce droit d'usage, parce qu'il est partagé, s'oppose au droit d'exclure et de réservation lié à la propriété traditionnelle ${ }^{26}$. Il s'agit bien de partage et non pas d'échange ${ }^{27}$, ce qui renvoie au fait que la chose commune est insusceptible d'échange mais bien un objet universel.

Cet usage, parce qu'il est commun, limite encore le pouvoir de l'homme sur cette chose ${ }^{28}$. En conséquence, «parce qu'elle est commune, une ressource ne peut supporter que des droits d'usage qui ne l'épuisent pas et laissent intact le droit d'usage d'autrui $»^{29}$.

Cet usage commun consacré par l'article 714 du Code civil est un usage trans-individuel (non exclusif), indivisible et non répartissable (entre les individus), trans-générationnel, dans la mesure où au-delà de l'individu, l'expression «tous » renvoie in fine à l'humanité ou l'espèce

\footnotetext{
${ }^{25}$ Cette obligation de chacun à exercer son droit d'usage devrait effectivement obéir à l'ancien standard du code civil français d'une gestion en bon père de famille et également aux exigences des articles 627 du Code civil relatif à une jouissance modérée, économe, en fonction des besoins, et 630 instaurant une obligation de ne disposer des fruits que pour ses propres besoins et ceux de sa famille.

${ }^{26}$ F. Girard, «La propriété inclusive au service des biens environnementaux Repenser la propriété à partir du bundle of rights », Cahiers Droit, Sciences \& Technologies, 6 | 2016, 185-236

${ }^{27}$ E. Leroy, «Sous les pavés du monologisme juridique la plage des communs et de néo communautés », in (dir. B. Parance et J. de Saint Victor) Repenser les biens communs, Paris, CNRS éd. 2014, p. 27

${ }^{28}$ Marie-Pierre Camproux Duffrène, «Plaidoyer civiliste pour une meilleure protection de la biodiversité ; la reconnaissance d'un statut juridique du règne animal », RIEJ, 2008, p. 1 à 28 et «Entre environnement per se et environnement pour soi ; la responsabilité civile pour atteintes à l'environnement », Env. et DD 2012, n 12, Étude $\mathrm{n}^{\circ} 14$ p. 13

${ }^{29}$ A. Gaonac'h, « Réparation du dommage écologique dans le droit de l'eau », RD rur. 2000, p. 279.
} 
humaine c'est-à-dire non seulement aux générations présentes mais également aux générations futures $^{30}$. Ainsi ce droit d'usage commun selon l'article 714 doit être reconnu comme perpétuel puisque lié à la chose commune vitale pour l'humanité. La chose commune doit pouvoir être transmise ce qui fait écho à la notion d'héritage auquel fait référence la théorie du patrimoine commun de l'humanité chère à Alexandre Kiss ${ }^{31}$. Cet usage commun a comme effet d'obliger à la transmission et donc à la conservation de la chose.

\section{Une obligation corrélative de conservation car sine qua non}

Cette obligation de conservation n'est pas prévue expressément dans la loi de 1803. Elle ne vient qu'en déduction de l'usage commun, c'est une conséquence implicite mais nécessaire $^{32}$. Ceci s'explique par le fait qu'à l'époque comme à celle du droit romain, au vu du bon état de l'environnement naturel et de l'abondance du vivant, les limites planétaires, la dégradation de la planète et des écosystèmes et la perte de biodiversité mises en lumière actuellement n'étaient pas envisagées. Aujourd'hui, cette obligation apparaît fondamentale aux tenants de ce statut de la chose commune, elle est la contrepartie sine qua non de cet usage commun. Elle devrait pour plus de garantie être précisée dans l'article 714 pour permettre une meilleure assise et au juge de s'en emparer ${ }^{33}$.

Le maintien de cet usage sur la chose commune «environnement naturel » est conditionnée par l'absence, ou plutôt l'arrêt, de pertes de substance et de fonctionnalité de celleci. Selon B. Jadot, pour que chacun puisse en jouir, il faut la conserver ${ }^{34}$. Ainsi, cette obligation de conservation qui porte sur la chose repose sur chaque bénéficiaire de cet usage à l'égard des co-usagers. L'usage de la chose commune n'étant pas détaché d'un droit de propriété il est tout à fait sensé que l'obligation de conservation pèse sur les bénéficiaires de l'usage. À ce propos,

\footnotetext{
${ }^{30} \mathrm{~V}$. Nos développements à propos de l'intérêt collectif environnemental. « Cette trans-individualité entre en résonance avec la qualification de l'environnement en chose commune (inappropriée et inappropriable) et la théorie adjointe selon laquelle l'homme a un droit d'usage partagé sur celle-ci et non un droit de propriété exclusive. Elle permet ainsi de préciser qu'en cas d'atteinte à l'environnement, l'intérêt collectif afférent défend des droits indivisibles, insusceptibles de faire l'objet d'une répartition entre les individus », M-P Camproux Duffrène « la représentation de l'intérêt collectif environnemental devant le juge civil : après l'affaire Erika et avant l'introduction dans le Code civil du dommage causé à l'environnement, préc.

${ }^{31}$ Alexandre-Charles Kiss, «La notion de patrimoine commun de l'humanité », in Recueil des cours de l'Académie de droit international (RCADI) 1982 tome 175 p. 99-256

${ }^{32}$ V. M.-A. Chardeaux, Les choses communes, LGDJ, 2006, p.334 et s. et notamment $n^{\circ} 323$ et 325.

${ }^{33}$ Il faut préciser que l'article 714 du Code civil n'a jamais servi de fondement à la jurisprudence française. Un arrêt de rejet et inédit de la Cour de Cassation en Chambre civile 1, du 25 février 2003, pourvoi n01-02.149 a retenu dans son attendu que «l'eau, fût-elle un bien commun, étant susceptible d'appropriation et pouvant être assimilée à une marchandise ». Mais sans que cette décision n'apporte à notre démonstration sauf à distinguer l'eau comme bien commun et la possibilité de vendre de l'eau assainie (https://www.legifrance.gouv.fr/affichJuriJudi.do?oldAction=rechExpJuriJudi\&idTexte=JURITEXT0000074586 07\&fastReqId=2074414734\&fastPos=6). M.-A Chardeaux dès 2006 encourageait cependant le juge à s'emparer de cet article en énonçant que « Toute personne qui méconnaitrait ce devoir commettrait une faute, engageant sa responsabilité civile délictuelle sur le fondement des articles 714 et 1382 du Code civil. »

${ }^{34}$ Benoit Jadot, «L'article 714 du Code civil et la protection de l'environnement », in F. Ost et S. Gutwirth, Quel avenir pour le droit de l'environnement?, Bruxelles, Pub. Faculté universitaires de Saint Louis 1996, p. 58 à 61
} 
P. Dardot et C. Laval écrivent que « la co-obligation des 'hommes du commun' est celle qui leur impose d'user de cet inappropriable de manière à le préserver et à le transmettre ${ }^{35}$.

Pour permettre la conservation dans toute son intégrité de la chose objet de droit et subséquemment du droit d'usage d'autrui, chaque usager doit non seulement ne pas dégrader la chose, mais également prendre toutes les mesures qui permettront aux co-usagers ou futurs usagers de jouir de la chose commune.

Et au regard de la singularité de l'objet sur lequel elle porte, cette obligation de conservation doit être particulièrement renforcée.

En effet, en miroir d'un droit d'usage vital et perpétuel, l'obligation de conservation doit être renforcée. Il est tout d'abord évident que cette obligation de conservation concerne le long terme, pour bénéficier non seulement aux générations actuelles mais également aux générations futures $^{36}$. En effet, cette conservation de la chose commune, si elle est vitale pour l'homme en tant qu'individu ou en tant qu'usager actuel, l'est également pour l'espèce humaine dépendante de cette chose avec laquelle il fait destin commun et donc pour les générations futures.

Cette obligation devrait dès lors empêcher en particulier toute atteinte irréversible nuisant à l'usage des générations futures en violation de l'article 714 du Code civil et répondre à la Charte de l'environnement selon laquelle « les choix destinés à répondre aux besoins du présent ne doivent pas compromettre la capacité des générations futures et des autres peuples à satisfaire leurs propres besoins ».

Dans cette perspective, le droit devrait sanctionner pénalement comme civilement, voire administrativement d'autant plus sévèrement les auteurs de dommages écologiques irréversibles (ce que ne fait pas le droit français aujourd'hui) et ce par tout moyen par application notamment des différents principes directeurs visant à la protection de l'environnement inscrits dans la Charte de l'environnement et l'article L110-1 I du Code de l'environnement.

\section{L'article 714 du Code civil à activer au regard des enjeux}

Au regard de l'urgence écologique proclamée par des scientifiques de toutes disciplines, il est temps d'activer le dispositif de l'article 714 du Code civil.

L'usage commun consacré par l'article 714 du Code civil peut tout d'abord être reconnu comme ayant une nature spécifique (A). Au regard de celle-ci, des mécanismes protecteurs de droit civil peuvent être mobilisés pour améliorer l'effectivité de cet article (B).

\footnotetext{
${ }^{35}$ P. Dardot et C. Laval, Commun. Essai sur la révolution au xxi siècle, la découverte, 2014, p. 283

${ }^{36}$ La notion d'autrui est alors interprétée largement pour englober non seulement les personnes existantes mais également les générations futures. V. M.-P. Camproux Duffrene «La représentation de l'intérêt collectif environnemental devant le juge civil : après l'affaire Erika et avant l'introduction dans le Code civil du dommage causé à l'environnement », préc.
} 


\section{A. Un usage commun comme un droit subjectif de nature complexe}

Cet usage commun à tous de l'article 714 est tout à fait spécifique en ce qu'il induit des prérogatives restreintes sur la chose commune et ce justement afin de la protéger d'un pouvoir trop grand, 1'homme étant alors considéré comme un prédateur plutôt que comme un bon gardien $^{37}$. Il est donc frontalement différent du droit de propriété. Dans ce sens, pour P. Dardot et C. Laval, «l'usage instituant les communs n'est pas un droit de propriété, il est la négation en acte du droit de propriété sous toutes ses formes parce qu'il est la seule forme de prise en charge de l'inappropriable $»^{38}$.

Les particularités de cet usage commun ont été relevées en ce qu'il est la traduction d'une superposition de liens. Cet usage peut être analysé au regard des grandes notions du droit privé $^{39}$. Il en sera tenté un décryptage juridique loin d'être achevé et sécurisé théoriquement ${ }^{40}$. Chaque proposition peut donner lieu à des débats passionnés. Il est proposé que cet usage commun soit qualifié de droit subjectif et commun (1), réel et autonome (2) fondamental car vital (3).

\section{Un droit subjectif et commun}

Il ne sera pas rentré dans les débats nombreux sur la notion de droit subjectif. Il faut bien sûr avoir en conscience la distinction entre intérêts et droits ${ }^{41}$ car si la notion d'intérêt collectif (au sens diffus) est admise en droit français ${ }^{42}$ celle de droit subjectif collectif (ou commun) est loin d'être évidente ${ }^{43}$. Certains prônent l'utilisation dans ce cas de la notion de privilège développée en droit anglo-saxon par Wesley Newcomb Hohfeld ${ }^{44}$ et développé par Yochai

\footnotetext{
${ }^{37}$ V. Les débats d'aujourd'hui sur la tragédie des communs décrite par de Garett Hardin et la résurgence des communs, notamment par S Gutwirth et I. Stengers

${ }^{38}$ P. Dardot et C. Laval, Commun. Essai sur la révolution au xxi ${ }^{e}$ siècle, Paris, La découverte, 2014, p 233 et s.

${ }^{39}$ V. Le précieux chapitre sur les droits subjectifs p. 144 à 208, de J. Rochfeld in Les grands notions du droit privé, Paris, PUF Thèmis droit, 2011

${ }^{40} \mathrm{Ce}$ décryptage est loin d'être aussi approfondi qu'il serait nécessaire. Et les propositions faites sont évidemment discutables et améliorables.

${ }^{41} \mathrm{~V}$. François Ost, Droit et intérêts : Entre droit et non-droit, l'intérêt : essai sur les fonctions qu'exerce la notion d'intérêt en droit privé, pub. Fac. Saint Louis 1990, 201 p. Voir aussi Thierry Léonard, Conflits entre droits subjectifs, libertés civiles et intérêts légitimes. Un modèle de résolution basé sur l'opposabilité et la responsabilité civile, Fac. Saint Louis 2004, http://hdl.handle.net/2078.3/168645

${ }^{42}$ V. I. Omaree et L. Sinopoli (dir. sc.), Les actions en justice au-delà de l'intérêt personnel, Dalloz Actes, 2014, Et Emmanuel Jeuland, « la notion d'intérêt comme moyen d'immixtion dans les rapports de droit », in La défense de l'intérêt général par les associations, intérêt général versus intérêts collectifs, LGDJ, coll. Grands colloques, 2015 p. 57 et s.

43 M.-P. Campoux-Duffrène, «Essai de dialectique sur une responsabilité civile en cas d'atteinte à l'environnement », Pour un droit économique de l'environnement, Mélanges en l'honneur de G. Martin, éd. Frison-Roche, 2013, p. 108. Et «Pour une approche socio-écosystémique de la dette écologique: une responsabilité civile spécifique en cas d'atteintes à l'environnement», éd.. VertigO. 2016, DOI: $10.4000 /$ vertigo. 17493

${ }^{44}$ W. N. Hohfeld, « Some fundamental Legal conceptions as Applied in Judicial Resasoning », Yale L.J. 23 (1913), p.16-59
} 
Benkler ${ }^{45}$. Cependant, cet usage peut être considéré comme constitutif d'un droit subjectif « reconnu par le droit objectif (art 714 du Code civil) envers tel ou tel avantage matériel ou moral légitime (usage) au profit d'un titulaire (l'homme) $»^{46}$. De plus, il peut être argué que le droit subjectif n'est pas forcément synonyme d'exclusivité. Et le fait que cet usage soit commun à tous et que donc l'intérêt en cause soit commun ne nous paraît pas en contradiction avec sa subjectivité. Ce serait nier la trans-individualité du collectif, du commun ${ }^{47}$. Il est possible d'y voir un droit du sujet qui soit commun c'est-à-dire trans-individuel et donc trans-subjectif.

\section{Un droit réel autonome $e^{48}$}

Le droit réel est considéré comme un pouvoir direct sur la chose sans intermédiaire et n'ayant pas besoin d'un tiers, d'une personne pour pouvoir s'exercer, il est opposable erga omnes c'est-à-dire que chacun doit le respecter

Le droit d'usage de l'article 714 du Code civil traduit le lien qu'a chaque homme directement avec la chose commune. Ce droit semble devoir être qualifié de réel même s'il est évidemment très lié au fait que chaque homme est en relation factuelle étroite avec chaque autre homme pour pouvoir bénéficier de 1 'accès à la chose ${ }^{49}$. Ce droit peut être considéré comme ayant une fonction hautement sociale fondamentale comme l'a d'une manière bien moindre le droit de propriété.

Une fois encore, il est possible d'avancer qu'un pouvoir direct sur la chose n'est pas synonyme d'un pouvoir exclusif sur la chose. Ce droit d'usage commun fondé sur l'article 714 du Code civil peut être qualifié de réel car direct, même si effectivement il n'est pas exclusif.

\footnotetext{
${ }^{45}$ Y. Benkler, «Between Spanish Huertas and the Open Road: a Tale of Two Commons? », in Gouverning Knowledge commons, B. Frischmann, M. Madison, K. Strandburg (dir.), Oxford University Press, 2014, p. 64., V. S. Broca, «Benkler Yochai », in Dictionnaire des communs, M. Cornu, F. Orsi, J. Rochfeld (dir.), PUF, 2017, p. 241

${ }^{46}$ J. Rochfeld in Les grands notions du droit privé, Paris, PUF Thèmis droit, 2011, p. 155

47 V. Alexandra Aragão «Les intérêts diffus, instruments pour la justice et la démocratie environnementale » et Maria José Azar-Baud «L'action de groupe, une valeur ajoutée pour l'environnement ? »», VertigO - la revue électronique en sciences de l'environnement [En ligne], Hors-série 22 |septembre 2015, mis en ligne le 10 septembre 2015, consulté le 16 avril 2018. URL: http://journals.openedition.org/vertigo/16284 ; DOI : $10.4000 /$ vertigo. 16284

${ }^{48}$ Il est alors intéressant de remarquer, en matière de troubles anomales du voisinage, une hésitation doctrinale similaire en ce qui concerne le fondement réel ou personnel de la responsabilité (Voir R. Libchaber, «Le droit de propriété, un modèle pour la réparation des troubles du voisinage », in Mélanges Christian Mouly, t. 1, Paris, Litec, 1998, p. 421).

${ }^{49}$ Une discussion pourrait avoir lieu sur la nature réelle ou personnelle de ce droit (V. aussi Y. Strickler, Droit des biens, LGDJ, Collection cours, 2017 p. 38 n $^{\circ} 12,13$ et 20 et sur les droits droits portant sur l'environnement ; J.C. Saint-Pau, «Le patrimonial et l'extrapatrimonial à l'épreuve des questions environnementales », Les notions fondamentales de droit privé à l'épreuve des questions environnementales, (dir. M. Mekki), Bruxelles, éd. Bruylant, Droit (s) et développement durable, 2016, p.104). Mais ce qualificatif paraît inadapté dans la mesure ou un droit personnel crée un lien entre une personne créancière et un autre débiteur qui donne un pouvoir d'exiger d'une autre personne qu'il accomplisse une prestation constituant l'objet de ce droit et que pour obtenir l'utilité de la chose, il faudrait passer par l'intermédiaire d'une personne qui fournirait cette utilité (V. J. Rochfeld, Les grandes notions du droit privé, Paris, éd. PUF, Thémis du droit 2011, p. 145 et s., notamment $\left.\mathrm{n}^{\circ} 156, \mathrm{n}^{\circ} 7\right)$. Il semble important de ne pas confondre le caractère social de la fonction d'un droit et sa nature réelle ou personnelle.
} 
Pour être précis, ce droit d'usage commun à tous ne doit pas être confondu avec un droit réel démembré. Il existe en tant que tel et non pas comme étant détaché ou autrement dit comme étant un démembrement du droit de propriété issu de l'article 544 du Code civil. Cet usage commun est prévu expressément, et de manière impérative, par l'article 714 dans son alinéa $1^{\text {er }}$ et il est indiqué à l'alinéa 2 de l'article 714 que ce sont des lois de police qui règlent la manière d'en jouir. Sa localisation dans le code civil le place en dehors des droits personnels en dehors des droits réels liés à la propriété dans les dispositions générales traitant des cas particuliers.

Il faut ajouter également que chacun des co-usagers de la chose commune est tenu d'une obligation d'assurer la pérennité de la chose commune per se mais également afin de respecter l'usage des autres et non pas, contrairement à l'usufruit, pour préserver le droit d'un propriétaire qui fait défaut ${ }^{50}$.

Ces différents éléments justifient que cet usage commun fondé sur l'article 714, et donc hors catégorie des droits portant sur les choses appropriées, soit reconnu comme un droit réel autonome $^{51}$, distinct du droit d'usage démembré du droit de propriétée ${ }^{52}$ ou de l'usufruit du livre 2 du Code civil ${ }^{53}$.

Il faut noter que le droit français paraît prêt à s'ouvrir à de nouvelles catégories de droit réel sui generis ${ }^{54}$. Et le droit d'usage commun de l'article 714 du Code civil peut être identifié comme distinct car imprescriptible, intransmissible, insaisissable, incessible, inaliénable ce qui le distingue des droits réels démembrés. Il devrait être reconnu comme tel puisque vital pour chacun et tous.

Ce droit réel autonome à forte fonction sociale a par conséquent par nature un fort potentiel de conflictualité.

Ainsi mis en lumière, cet article 714 doit pouvoir être sanctionné par le juge. En tant que droit réel, chacun doit pouvoir le défendre devant le juge en cas d'empiètement d'un tiers. Et parce que ce droit est également commun et qu'il empêche d'avoir un droit exclusif sur la chose au sein de la communauté, il doit permettre d'arguer devant le juge d'un mauvaise exercice de

\footnotetext{
${ }^{50}$ A. Sériaux, «La notion de choses communes, nouvelles considérations juridiques sur le verbe avoir », in J.-Y. Chérot et al., Droit et environnement. Propos pluridisciplinaires sur un droit en construction, Aix-Marseille, éd. PUAM 1995, p. 35.

${ }^{51}$ V. également Fabien Girard, «La propriété inclusive au service des biens environnementaux Repenser la propriété à partir du bundle of rights », Cahiers Droit, Sciences \& Technologies, 6|2016, 185-236. Pour qui « Il faudra à un moment ou à un autre que soit discutée la possibilité de créer des droits réels démembrés suffisamment longs (au-delà donc du terme trentenaire ou quasi séculaire qui paraît souvent s'imposer aux esprits pour assurer une protection effective de l'environnement ».

${ }^{52}$ Même si certaines règles applicables au droit d'usage démembré peuvent être adaptées dans la relation entre l'individu et la chose commune.

${ }^{53}$ V. Civ. 3, 31 octobre 2012, $\mathrm{N}^{\circ} 16-304$, Bull. ${ }^{\circ} 159$ L. d'Avout et B.Mallet-Bricout, « De l'autonomie, de la durée et des causes d'extinction des droits réels de jouissance spéciale », D. 2017. 134.

${ }^{54}$ Nicolas Kilgus, « Droit réel de jouissance spéciale et perpétuité : une nouvelle étape ?, com. De Civ. 3, 7 juin 2018, n¹7-17.240, D. 19 juin 2018, flash, https://www.dalloz-actualite.fr/flash/droit-reel-de-jouissance-specialeet-perpetuite-une-nouvelle-etape?, faisant suite à Civ. 3, 31 octobre 2012, N¹6-304, Bull. $\mathrm{n}^{\circ} 159$ L. d'Avout et B.Mallet-Bricout, « De l'autonomie, de la durée et des causes d'extinction des droits réels de jouissance spéciale », D. 2017. 134
} 
ce droit (et de son obligation corrélative de conservation), d'un dépassement de ce droit par un co-usager, voire d'un trouble anormal au droit d'usage de la chose commune ${ }^{55}$.

\section{3. un droit fondamental car vital}

Ce droit d'usage étant vital tant physiquement que mentalement et socialement, il pourrait être considéré comme un droit fondamental rattaché à la personne et indisponible.

Cette catégorie des droits fondamentaux est en pleine extension et compose une classe spéciale des droits subjectifs à laquelle on attache une valeur et une force supérieure aux droits subjectifs ordinaires ${ }^{56}$. Ce critère substantiel du droit et de la valeur qu'il traduit ou de sa valeur matricielle au regard d'autres droits parait s'adapter au droit d'usage commun de l'article 714 du Code civil.

Il semble possible de qualifier cet usage commun de droit fondamental englobant le fait qu'il soit à la fois un droit réel fondamental (car portant sur une chose indispensable à la vie ce qui permet de ne pas occulter l'importance de la chose commune per se) et un droit fondamental rattaché à la personne (puisqu'une personne dépourvue de cet usage est vouée à la mort, ce qui permet de lui attribuer les caractéristiques liées à son indisponibilité). Son rapprochement avec l'article $1^{\text {er }}$ de la Charte de l'environnement ne peut que confirmer cette analyse.

Il faut noter que les droits relatifs aux beni communi en Italie sont qualifiés de droits fondamentaux rattachés à la personne par la commission Rodota ${ }^{57}$. De même, pour B. Jadot, le droit d'usage commun de la chose commune de l'article 714 du Code civil belge est un droit rattaché à la personne : chaque être humain a un droit de ne pas être privé des choses vitales et «priver quelqu'un de la jouissance des choses communes c'est le priver de ce qui est indispensable à la vie $»^{58}$.

In fine, ce droit d'usage commun peut être qualifié de droit réel autonome ne serait-que par sa localisation dans le Code civil, mais peut être qualifié également de droit fondamental rattaché à la personne pour répondre à sa singularité et son importance pour notre survie.

\footnotetext{
${ }^{55} \mathrm{Il}$ est intéressant de relever non pas à propos de ce droit d'usage commun tiré de l'article 714 du code civil mais du droit de chacun à avoir un environnement équilibré et respectueux de la santé de l'article 1 articulé avec le devoir de tous de protéger l'environnement fondé sur de l'article 2 de la Charte de l'environnement, que ceux-ci tels qu'interprétés par la décision du conseil constitutionnel de 8 avril 2011 semblent former une obligation qui puisse être invoquée devant le juge sur le fondement de la responsabilité juridique et qu'il soit possible d'agir en justice en cas de manquement.(V Décision n 2011-116 QPC du 8 avril 2011 | Conseil constitutionnel, M. Michel $\mathrm{Z}$. et autre [Troubles du voisinage et environnement], https://www.conseilconstitutionnel.fr/decision/2011/2011116QPC.htm )

${ }^{56}$ V. J. Rochfeld, Les grandes notions du droit privé, préc., p. 164

57 Stefano Rodotà, «Vers les biens communs. Souveraineté et propriété au XXI ${ }^{\mathrm{e}}$ siècle », Tracés. Revue de Sciences humaines [HS 16 |2016, mis en ligne le 01 janvier 2017.

${ }^{58} \mathrm{~B}$. Jadot, « l'environnement n'appartient à personne et l'usage qui en est fait est commun à tous », in Quel avenir pour le droit de l'environnement? (dir. F. Ost et S. Gutwirth), Bruxelles, Publ. Facultés universitaires de Saint Louis, Bruxelles, 1996, p. 56.
} 
La qualification de cet usage commun en un droit subjectif se prolonge par l'attribution de prérogatives juridiques permettant de l'exercer, notamment par le biais d'une action en justice permettant l'effectivité de l'article 714 du Code civil.

\section{B. La mobilisation de mécanismes civilistes à l'appui de l'effectivité de l'usage commun de la chose commune}

Cet usage commun qui oblige les co-usagers au respect de la chose et envers ses pairs peut être activé en utilisant pour ce faire des mécanismes de droit civil parce que ce lien juridique est consacré par le Code civil et parce qu'il peut s'appuyer sur l'alinéa 2 de l'art. 714 par renvoi à des lois de police qui règlent la manière d'en jouir ${ }^{59}$. Il est possible de se référer à l'arsenal du droit pénal de l'environnement, au aux dispositifs du Code de l'environnement qui peuvent être interprétés comme des textes d'application de cette jouissance de la chose commune. Il peut également être fait référence aux articles du Code civil régissant spécifiquement les eaux ou les animaux.

Mais indépendamment de l'alinéa 2, l'alinéa 1er consacrant un usage commun autonome applicable en l'occurrence à l'environnement (1), celui-ci doit pouvoir être défendu devant le juge civil (2).

\section{Un usage commun environnemental à défendre}

L'usage commun de l'article 714 du Code civil appliqué dans le domaine de l'environnement peut être renforcé par la Charte de l'environnement, laquelle appartient au bloc de constitutionnalité ${ }^{60}$. En particulier l'article $1^{\text {er }}$ de la Charte vient en appui au regard de son contenu ; « chacun a droit à un environnement équilibré et respectueux de la santé ».

Cet article $1^{\text {er }}$ peut s'articuler avec l'art 714 du Code civil. En effet, dans les deux textes l'objet concerné est l'environnement, même si cette qualification de l'article 714 du Code civil peut concerner d'autres entités que l'environnement. Et si le premier met l'accent sur l'homme comme sujet du droit à l'environnement, et le second porte plutôt sur le lien entre la chose commune «environnement naturel » et l'homme sujet de droit via l'usage commun, dans les deux l'objectif est bien d'améliorer la protection de la chose qualifiée de chose commune pour l'article 714, l'environnement en l'espèce et l'environnement dans la Charte de l'environnement.

La formulation de l'article 1 de la Charte est moins simple qu'il n'y parait. Tout d'abord, il faut relever que cet article reprend une formule classique «chacun a droit à » que nous retrouvons à l'article 9 du Code civil (« chacun a droit au respect de sa vie privée ») ou l'article

\footnotetext{
${ }^{59} \mathrm{~V}$. Les développements toujours actuels sur ce point de B. Jadot, in « L'article 714 du Code civil et la protection de l'environnement », préc. p.66 et s.

${ }^{60}$ Yann Aguila, La valeur constitutionnelle de la Charte de l'environnement, Conclusions sur Conseil d'Etat, ass., 3 octobre 2008, Commune d'Annecy, req. n 297931, RFDA 2008, p. 1147
} 
16-1 («chacun a droit au respect de son corps »). Il peut être précisé ensuite que cet article permet de fonder un droit à agir en justice discrètement consacré par une décision 2011-116 QPC du Conseil Constitutionnel du 8 avril $2011^{61}$. Il faut pour finir remarquer une différence fondamentale qui peut illustrer ce lien avec l'article 714 et relayer cette idée de communalité. En effet, selon l'article $1^{\text {er }}$ de la charte, chacun n'a pas droit à son environnement ou un environnement qui soit respectueux de sa santé. Il ne s'agit pas d'un droit personnalisé auquel chacun peut renoncer mais d'un droit à la fois fondamental et de caractère commun, indivisible. Ainsi est écrit l'article $1^{\text {er }}$ de la Charte : "Chacun a droit à un environnement équilibré et respectueux de la santé ». Est retrouvée cette relation complexe de l'article 714 d'un lien portant sur la chose mais qui relie aussi chacun à tous pour en faire un droit d'usage commun. Autrement dit, ce qui est mis en exergue autant dans l'article $1^{\text {er }}$ de la Charte que dans l'article $714 \mathrm{du}$ Code civil ce n'est pas l'identification d'un lien juridique exclusif entre la personne et la chose mais un lien diffus ${ }^{62}$ des hommes sur cette même chose « environnement ».

Cet usage commun peut être défendu en justice.

\section{Un droit d'action en justice pour défendre cet usage commun}

Il est juridiquement admis qu'une personne juridique puisse agir en justice au nom d'un intérêt collectif $^{63}$. Soit dans le cadre d'une action populaire qui n'existe pas (encore ?) ${ }^{64}$ en France mais dans d'autres pays notamment au Portugal et les pays lusophones. Soit dans le cadre d'une action en défense d'un intérêt collectif qui existe d'ores et déjà en France en matière syndicale, concernant l'intérêt des consommateurs et plus récemment pour le préjudice écologique au regard de la formulation de l'article 1248 du Code civil quant aux titulaires de l'action civile en réparation de celui-ci. Que ce représentant fasse partie lui-même de ce collectif n'amoindrit pas la dimension collective de l'intérêt représenté.

Juridiquement ce droit d'usage nous oblige envers autrui ${ }^{65}$. Il peut alors fonder une action en respect de ce droit fondamental ou l'engagement de la responsabilité civile en cas de

\footnotetext{
${ }^{61}$ Décision $n^{\circ}$ 2011-116 QPC du 8 avril 2011 | Conseil constitutionnel, M. Michel Z. et autre [Troubles du voisinage et environnement], https://www.conseil-constitutionnel.fr/decision/2011/2011116QPC.htm, F.-G. Trébulle, Le Conseil constitutionnel, l'environnement et la responsabilité : entre vigilance environnementale et préoccupation : RD imm. 2011, p. 369

${ }^{62} \mathrm{~V}$. Alexandra Aragão «Les intérêts diffus, instruments pour la justice et la démocratie environnementale », préc. et Maria José Azar-Baud «L'action de groupe, une valeur ajoutée pour l'environnement ? »», VertigO - la revue électronique en sciences de l'environnement [En ligne], Hors-série 22 | septembre 2015, mis en ligne le 10 septembre 2015, consulté le 16 avril 2018. URL: http://journals.openedition.org/vertigo/16284 ; DOI : $10.4000 /$ vertigo. 16284

${ }^{63}$ M-P Camproux Duffrène «L'accès au juge civil français en cas d'atteintes à l'environnement : une diversité d'actions pour répondre à la diversité des préjudices ", Le droit d'accès à la justice en matière d'environnement, (dir. J. Bétaille), Toulouse, éditeur les presses de l'UFR de l'Université Toulouse 1 Capitole, LGDJ Lextenso, 2016, p. 203 à 224.

${ }^{64}$ L'article 1248 du Code civil énumère un certain nombre d'entités juridiques aptes à agir mais laisse la voie ouverte à une généralisation de l'action du fait de sa formulation qui introduit une énumération par «toute personne ayant qualité et intérêt à agir».

${ }^{65}$ Il est écrit dans l'article 4 de la Déclaration des Droits de l'Homme et du Citoyen de 1789 que «La liberté consiste à pouvoir faire tout ce qui ne nuit pas à autrui : ainsi, l'exercice des droits naturels de chaque homme n'a
} 
manquement au droit d'usage d'autrui ou de dépassement de son propre droit pour mettre fin à la cessation de l'illicite ou pour réparer le dommage subséquent. Il pourra en effet être invoqué une atteinte, un empiètement au droit indépendamment de tout préjudice ${ }^{66}$. Il sera évidemment plus facile d'agir en cas de préjudice et ce sera le cas pour le préjudice écologique sur le fondement de la responsabilité juridique (pour l'instant civile) de l'usager.

En France, l'action en réparation du préjudice écologique introduite dans le Code civil par la loi du 8 août 2016 peut être considérée comme une action en défense d'un intérêt collectif environnemental. La question aujourd'hui qui se pose en France est de savoir qui peut défendre cet intérêt collectif environnemental. L'article 1248 du Code civil tel qu'il est rédigé pourrait ouvrir, en dehors des personnes expressément énumérées dans le texte et donc habilitées légalement à agir, la voie à une action populaire ou universelle. En effet, l'article énonce que « l'action en réparation du préjudice écologique est ouverte à toute personne ayant qualité et intérêt à agir », cette formulation laisse place à une possible interprétation large des juges ${ }^{67}$.

À noter que la définition du préjudice écologique ne choisissant pas entre approche écocentrée ou approche anthropocentrée, regroupe non seulement les atteintes aux éléments ou aux fonctions des écosystèmes (environnement per se) mais également les bénéfices collectifs tirés par l'homme de l'environnement ${ }^{68}$. Ce non-choix permet ainsi la prise en compte non seulement des atteintes à la chose commune «environnement » per se mais également des atteintes à l'usage commun des hommes sur l'environnement et permet en cas d'atteinte à l'environnement le respect du dispositif et des objectifs de l'article 714 du Code civil.

Cette introduction dans le Code civil d'une action spécifique en réparation du préjudice écologique pour une mise en œuvre adaptée ne repose pas seulement sur la définition du préjudice écologique mais de manière concomitante sur la désignation des titulaires et des modalités de réparation originales.

Quant au titulaire, l'article 1248 du Code civil $^{69}$ énumère un certain nombre d'entités juridiques aptes à agir représentant pour certains l'intérêt général qu'il y a à protéger l'environnement inscrit à l'article L110-1 du Code de l'environnement. Il désigne également et surtout pour notre démonstration les associations pour la protection de l'environnement qui, quant à elles, exercent « les droits reconnus à la partie civile en ce qui concerne les faits portant un préjudice direct ou indirect aux intérêts collectifs qu'elles ont pour objet de défendre » selon

de bornes que celles qui assurent aux autres Membres de la Société la jouissance de ces mêmes droits. Ces bornes ne peuvent être déterminées que par la Loi ».

${ }^{66} \mathrm{~V}$. Les actions en cas d'atteinte à la vie privé ou au droit de propriété (et la jurisprudence sur l'empiètement imposant la destruction de la construction sans nécessité de préjudice).

${ }^{67}$ M.-P. Camproux Duffrène, « L'accès au juge civil français en cas d'atteintes à l'environnement : une diversité d'actions pour répondre à la diversité des préjudices ", Le droit d'accès à la justice en matière d'environnement, dir. J Bétaille, éditeur les presses de l'UFR de l'Université Toulouse 1 Capitole, LGDJ Lextenso, 2016, p.203 à 224

${ }^{68}$ Article 1247 du Code civil «Est réparable, dans les conditions prévues au présent titre, le préjudice écologique consistant en une atteinte non négligeable aux éléments ou aux fonctions des écosystèmes ou aux bénéfices collectifs tirés par l'homme de l'environnement.».

${ }^{69}$ Art. 1248 du Code civil «L'action en réparation du préjudice écologique est ouverte à toute personne ayant qualité et intérêt à agir, telle que l'Etat, l'Agence française pour la biodiversité, les collectivités territoriales et leurs groupements dont le territoire est concerné, ainsi que les établissements publics et les associations agréées ou créées depuis au moins cinq ans à la date d'introduction de l'instance qui ont pour objet la protection de la nature et la défense de l'environnement» 
l'article L 142-2 du Code de l'environnement. Ces associations, en attendant une action populaire ou action universelle ${ }^{70}$ peuvent donc être les représentants de cet intérêt collectif et de ce droit en commun sur la chose commune.

Quant aux modalités de réparation de ce préjudice écologique, la loi de 2016 a modifié les règles de réparation civile du préjudice écologique afin de s'adapter à la singularité du préjudice concerné. En effet, l'article 1249 du Code civil ${ }^{71}$ prévoit de privilégier la réparation en nature et en cas de réparation par indemnisation subsidiaire prévoit l'attribution des sommes soit au titulaire de l'action s'il a les moyens techniques et humains de les affecter à la réparation de l'environnement (sic) soit à l'État qui se voit imposer l'affectation de ces sommes à cette même réparation. Ces modalités de réparation en nature permettent non seulement la réparation des dégradations ou atteintes faites à la chose commune, mais elles peuvent aussi tendre concomitamment à un rétablissement des divers usages humains ${ }^{72}$.

Ainsi, en matière environnementale, la responsabilité civile peut avoir comme finalité la restauration des équilibres lésés et la réparation des préjudices occasionnés par ce déséquilibre.

La lecture de cet article 714 et de sa mise en œuvre peut servir à l'intégration de l'environnement dans cette approche des communs dans la mesure où cette chose commune dans sa globalité n'est pas soumise au marché et échappe au contrôle de l'État.

\section{III- Une articulation de l'article 714 du Code civil avec les communs}

L'appréhension de l'environnement comme chose commune soumise à ce régime d'usage commun avec obligation de conservation semble pouvoir servir la conception de communs naturels distinguant les communs universels soumis à ce régime matriciel et les communs secondaires spatialisés (B). Cependant, cette approche repose sur une construction juridique qu'il faut expliciter. Comment concrètement est-il possible de raisonner au niveau global (environnement naturel) alors que l'homme n'accède lui dans les faits qu'à des spécimens ou des parcelles ou portions et donc des composantes ou productions de l'environnement naturel que l'homme peut utiliser, exploiter, prélever et/ou s'approprier, voire détruire ? Il faut donc étudier au préalable quelles peuvent être les conséquences juridiques de l'application de

\footnotetext{
${ }^{70}$ Véronique Jaworski, «Les représentations multiples de l'environnement devant le juge pénal : entre intérêts général, individuel et collectif », VertigO - la revue électronique en sciences de l'environnement [En ligne], Horssérie $22 \mid$ septembre 2015, mis en ligne le 10 septembre 2015, consulté le 16 avril 2018. URL: http://journals.openedition.org/vertigo/16272 ; DOI : 10.4000/vertigo.16272

71 Art. 1249 du Code civil «La réparation du préjudice écologique s'effectue par priorité en nature./ En cas d'impossibilité de droit ou de fait ou d'insuffisance des mesures de réparation, le juge condamne le responsable à verser des dommages et intérêts, affectés à la réparation de l'environnement, au demandeur ou, si celui-ci ne peut prendre les mesures utiles à cette fin, à l'Etat./L'évaluation du préjudice tient compte, le cas échéant, des mesures de réparation déjà intervenues, en particulier dans le cadre de la mise en ouvre du titre VI du livre Ier du Code de l'environnement. »

${ }^{72}$ Il est alors intéressant de remarquer qu'en matière de troubles anormaux du voisinage, la sanction du juge comporte souvent des mesures de cessation de l'illicite qui peuvent être très proche des mesures de réparation en nature par équivalent. (V. la thèse de M.-E. Roujou de Boubée, Essai sur la notion de réparation, LGDJ, 1974).
} 
l'article 714 du Code civil. Pour ce faire, nous nous contenterons d'approfondir le cas de la biodiversité (A).

\section{A. Les effets de l'application de l'article 714 du Code civil à la biodiversité}

La biodiversité est définie maintenant dans le Code de l'environnement ${ }^{73}$. Il est possible de qualifier celle-ci de chose commune. Il est cependant alors difficile de faire le lien avec la possibilité de vendre des légumes ou un troupeau, pêcher des poissons en eau libre, arracher une vigne pour y construire une maison ou marcher sur la pelouse alors que ces éléments font partie logiquement de la biodiversité qu'il est urgent de préserver au regard de son déclin toujours plus rapide.

Des explications sont possibles, elles s'appuient sur des constructions juridiques préexistantes : l'universalité de fait (1), la distinction fruits-produits (2) et la règle selon laquelle l'accessoire suit le principal (3). L'utilisation de ces dernières au bénéfice de l'environnement peut néanmoins aboutir à des changements de perspectives.

\section{La chose commune, universalité de fait}

La chose commune peut être, à l'image d'un fonds de commerce ou d'un portefeuille de valeurs mobilières, une universalité de fait. Cette qualification est définie selon R. Libchaber comme un agrégat de choses, corporelles ou incorporelles, dont une certaine cohérence d'ensemble justifie l'émergence en une totalité ${ }^{74}$. Elle consiste à réunir divers éléments sous un même régime juridique pour les traiter dans une perspective unique et une affectation commune soumise à des règles qui lui sont propres ${ }^{75}$.

Ainsi, la biodiversité est composée de différents éléments qui peuvent être traités comme une unité ne serait-ce que par leurs interactions alors qu'ils ressortissent à des catégories juridiques différentes. Ses composantes peuvent appartenir en effet à des catégories juridiques différentes et obéir en conséquence à des régimes différents. La qualification d'universalité de fait permet d'articuler de manière cohérente le statut de chose commune avec la diversité des régimes juridiques applicables aux individus ou à certaines parties de cette entité globale. Ainsi la biodiversité considérée comme une res communis, les éléments qui en font partie peuvent être des res nullius ${ }^{76}$ donc susceptibles d'être appropriées par occupation (ex : poissons en eau

\footnotetext{
${ }^{73}$ La loi n ${ }^{\circ}$ 2016-1087 du 8 août 2016 pour la reconquête de la biodiversité, de la nature et des paysages vient en effet d'intégrer une définition de la biodiversité dans le Code de l'environnement dans son article L110-1 I alinéa $3:$ : On entend par biodiversité, ou diversité biologique, la variabilité des organismes vivants de toute origine, y compris les écosystèmes terrestres, marins et autres écosystèmes aquatiques, ainsi que les complexes écologiques dont ils font partie. Elle comprend la diversité au sein des espèces et entre espèces, la diversité des écosystèmes ainsi que les interactions entre les organismes vivants».

${ }^{74}$ V. R. Libchaber, Répertoire de droit civil, Biens, Paris, Dalloz - septembre 2009 (mise à jour : juin 2010).

${ }^{75}$ V. Y. Strickler sur la notion d'universalité de fait, in Droit des biens, LGDJ, Lextenso, Coll. Cours, 2017, n ${ }^{\circ}$ 281 et $\mathrm{s}$.

${ }^{76}$ V. M. Rémond-Gouilloud, « Ressources naturelles et choses sans maître », D.1985, chron. p. 27.
} 
libre, gibier), des res propriae (ex : spécimens d'espèces domestiques) ou des res communes (spécimens d'espèces protégés ou les espèces en tant que telles).

La nature juridique des individus, des spécimens, portions, peut, du fait de cette appartenance, évoluer en raison de ce rattachement à la chose commune.

\section{La distinction fruit-produit de la chose commune}

La qualification de la biodiversité en chose commune permet de régir les relations entre l'homme et les éléments matériels de la chose. Ainsi l'homme qualifié par l'article 714 d'usager de la chose ne peut en conséquence juridiquement percevoir que les fruits de la chose et non ses produits. Ce régime juridique laisse à chacun un accès à des éléments vitaux, mais évite en parallèle toute atteinte à la substance de la biodiversité et à son bon fonctionnement. Chaque usager doit limiter les prélèvements et ne les réaliser que dans la mesure où ils ne portent que sur des spécimens qualifiables de fruits c'est-à-dire dont le prélèvement n'aura pas d'impact sur la substance même de la chose, le bon fonctionnement de l'écosystème. Si ce prélèvement entame ou dégrade la substance de la chose, alors l'élément n'est pas un fruit mais un produit que l'usager ne peut percevoir ou s'approprier. Ce dernier ne peut alors en faire qu'un usage modéré afin de permettre la conservation de l'intégrité de la chose.

Cette distinction de nature entre fruits et produits permet de comprendre pourquoi certains éléments de la biodiversité peuvent être appropriés et d'autre pas. Les spécimens d'espèces protégées ne sont pas appropriables pour la raison simple que leur prélèvement porte atteinte à la substance de la biodiversité et à son bon fonctionnement. Les spécimens des espèces protégées ne sont pas des rei nulli appropriables par occupation mais des produits de la chose commune régis par le régime de la chose commune. En revanche, une cueillette de marguerites dans un pré ou l'abattage d'un sanglier ou d'un mouton ne nuit pas à la chose-environnement et relève de son usage. Ces marguerites, ce lièvre ou ce mouton sont alors des fruits que l'usager de la chose commune peut s'approprier ${ }^{77}$.

Cette distinction entre fruit et produit pour les éléments détachables doit limiter l'appropriation aujourd'hui quasi automatique des éléments prélevés de la biodiversité et devrait aboutir à la réduction des spécimens susceptibles d'appartenir à la catégorie des res nullius. D'ores et déjà d'ailleurs il faut constater que le chasseur ou le pêcheur est limité dans ses prélèvements ${ }^{78}$.

\footnotetext{
${ }^{77}$ Il est vrai que le prélèvement de cet animal, même s'il correspond au droit d'usage de chacun (usage modéré et pour ces besoins) de l'article 714 du Code civil peut aboutir, en cas d'un trop grand nombre d'usagers, à une atteinte à la substance de la chose. Une régulation de cet accès à la chose doit donc être mise en place.

${ }^{78}$ Les critères de cette distinction juridique sur les éléments environnementaux sont au nombre de deux. Le premier est classique est repose sur le fait que leur prélèvement altère ou non la substance même de la chose dont ils sont issus, au regard de leur abondance ou de leur rareté. Le second est plus récent et a trait à leur importance pour le bon fonctionnement de l'écosystème ou les équilibres biologiques ou le maintien de la diversité biologique et si ce prélèvement porte atteinte à la variabilité et l'adaptabilité des organismes vivants et donc au maintien de la diversité biologique.
} 
Un prélèvement excessif, autrement dit une consommation excessive et non maîtrisée ${ }^{79}$ (par exemple en cas de surpêche entrainant disparition de l'espèce et appauvrissement de la biodiversité), devrait pouvoir entraîner une requalification de l'élément prélevé de fruit en produit. Son prélèvement devrait dès lors aboutir à une mise en cause de la responsabilité de l'auteur pour atteinte à la chose commune elle-même, pour dépassement de son droit qui n'est que d'usage et atteinte au droit d'usage des co-usagers via le mécanisme de la responsabilité civile ${ }^{80}$.

\section{L'application de la règle selon laquelle l'accessoire suit le principal}

Dans la mesure de l'identification de la biodiversité comme chose commune, la règle selon laquelle l'accessoire suit le principal peut permettre de comprendre la dissociation intéressante qu'il est possible de faire entre sol et biodiversité.

La définition de la biodiversité issue de l'alinéa 3 de l'article L110-1 I du Code de l'environnement comprend «la diversité au sein des espèces et entre espèces, la diversité des écosystèmes ainsi que les interactions entre les organismes vivants ». Selon l'alinéa $1^{\text {er }}$ de ce même article, la biodiversité fait partie du patrimoine commun de la nation. Cependant dans l'alinéa 2 le sol est exclu du patrimoine commun de la nation puisqu'il il y est indiqué expressément qu'il ne fait que concourir à la constitution de ce patrimoine. Le sol doit donc être dissocié des écosystèmes terrestres, ce qui peut être difficile à comprendre, le sol étant en lui-même un écosystème.

Cela étant cette dissociation peut se comprendre si l'on change d'approche. En effet, le sol fait l'objet de propriété (et les territoires de souveraineté de l'État). Le Code civil qualifie le fonds de terre d'immeuble par nature selon l'article 518 du Code civil ${ }^{81}$ et donc une res propria.

L'application de l'article 714 à la biodiversité peut permettre de trouver une articulation possible et des effets positifs à cette dissociation entre la biodiversité et les écosystèmes régies par le Code de l'environnement et le sol ou fonds de terre régi par les articles 518 et s. du Code civil.

La qualification de la biodiversité par le biais de l'article 714 du Code civil de chose commune lui donne une existence civiliste. Son identification peut changer ou rectifier certaines interprétations faites du Code civil.

Notamment nous pouvons réinterpréter certaines règles d'acquisition de la propriété notamment celles reposant sur la règle selon laquelle l'accessoire suit le principal.

\footnotetext{
${ }^{79}$ V. S. Drapier «Quel régime de responsabilité civile pour les choses communes endommagées ? , RJE 4/2016 p. 691

${ }^{80}$ M-P Camproux Duffrène, «Pour une approche socio-écosystémique de la dette écologique : une responsabilité civile spécifique en cas d'atteintes à l'environnement », VertigO - la revue électronique en sciences de l'environnement [En ligne], Hors-série 26 | septembre 2016, mis en ligne le 09 septembre 2016, consulté le 01 mars 2018. URL : http://journals.openedition.org/vertigo/17493 ; DOI : 10.4000/vertigo.17493

${ }^{81}$ Article 518 du Code civil : «les fonds de terre (...) sont immeubles par nature ».
} 
En effet, la biodiversité et ses composantes ne sont pas forcément rattachées juridiquement au sol comme il est souvent présumé et donc appropriés ou appropriables par occupation. Les spécimens d'espèces végétales ou animales peuvent ne pas être forcément perçus comme un accessoire à la terre et donc immeuble par incorporation ou comme faisant partie du dessus ou du dessous du sol approprié. Et à relire attentivement l'article $552 \mathrm{CC}$ dans son ensemble, il est possible de ne rattacher à la propriété du sol en tant que dessus ou dessous que le construit ou les plantations (liés à l'activité humaine et non à l'activité des processus biologiques et des écosystèmes ou des interactions entre organismes vivants $)^{82}$.

Si ces organismes vivants ou écosystèmes locaux ne sont pas l'accessoire du sol approprié, ils peuvent être, en revanche, rattachés à la biodiversité comme étant l'accessoire ou comme partie intégrante de la biodiversité qualifiée de chose commune. Dans ce cas, en tant qu'accessoire de cette chose commune ou partie de la chose commune, ils vont suivre son régime sauf à distinguer encore dans ces éléments les fruits des produits.

Ainsi les spécimens d'une espèce végétale ou animale sur le sol ne sont pas l'accessoire du sol mais l'accessoire de la biodiversité et, selon qu'ils sont fruits ou produits, ils pourront faire l'objet de prélèvement ${ }^{83}$ par l'homme usager de la biodiversité, éventuellement propriétaire du sol de surcroit.

En tant qu'accessoires de la biodiversité, ces spécimens peuvent voir leur régime juridique subir des adaptations en conséquence de leur rattachement à la biodiversité. Une population d'une catégorie d'arbres peut, du fait de sa rareté ou de son importance pour le bon fonctionnement de l'écosystème avec lequel elle interagit, ne pas être qualifiée de fruits et donc de bien approprié par le propriétaire du sol. Elle peut au contraire être identifiée comme un produit de la chose commune dont le prélèvement porte atteinte à la substance de la chose commune et d'accessoire à la chose commune et donc dans les deux cas devant suivre le régime de la chose commune c'est-à-dire être inappropriée et inappropriable. Cette population devrait alors vraisemblablement être qualifiée de spécimens d'une espèce protégée régie par le Code de l'environnement, ce régime de protection pouvant être identifié comme l'une des règles de police réglant la jouissance de la chose commune auquel fait référence l'alinéa 2 de l'article 714 du Code civil.

Dans cette construction juridique, il faut voir que, plus la biodiversité est en danger, plus les spécimens relèvent de la catégorie du produit et de celles des espèces protégées. Et donc moins le propriétaire aura de droit sur les productions spontanées de la biodiversité, ce qui est l'apport et les conséquences recherchées du régime juridique de la chose commune selon la lecture actualisée de l'article 714 du Code civil.

La question qui peut se poser est de savoir ce qu'il reste au propriétaire du sol. Il lui reste d'une part la propriété d'une assiette sol et des productions liés à son activité (plantations,

\footnotetext{
${ }^{82}$ Article 552 du Code civil «La propriété du sol emporte la propriété du dessus et du dessous. Le propriétaire peut faire au-dessus toutes les plantations et constructions qu'il juge à propos, sauf les exceptions établies au titre "Des servitudes ou services fonciers" ".

"Il peut faire au-dessous toutes les constructions et fouilles qu'il jugera à propos, et tirer de ces fouilles tous les produits qu'elles peuvent fournir, sauf les modifications résultant des lois et règlements relatifs aux mines, et des lois et règlements de police. "

${ }^{83}$ Et en tant que biens peuvent alors faire l'objet d'échanges marchands.
} 
constructions) et d'autre part éventuellement le privilège de l'usager gardien de ce qui est sur son sol et qui n'est pas lié à son activité de production ou de construction. En tant qu'usager gardien ou dépositaire, il doit alors exercer une jouissance précautionneuse de ces éléments au regard de celle qu'ont également ses co-usagers. Les techniques aujourd'hui mises en place comme l'obligation réelle environnementale ou la fiducie environnementale peuvent permettre cette gestion pour tous.

Nous sommes déjà dans ces derniers développements pour partie dans une approche intégrant celle des communs. Mais c'est l'ensemble de la réflexion menée qui peut apporter à la théorie des communs en matière environnementale.

\section{B. L'apport de l'article 714 du Code civil à la construction juridique des communs naturels}

Nous ne parlerons ici que des communs en matière environnementale que nous désignerons sous le terme de communs naturels.

Il faut distinguer la chose commune environnementale que nous venons de présenter de ce qui est identifié comme communs. Selon P. Dardot et C. Laval «On parlera des «Communs » pour désigner non pas ce qui est commun, mais ce qui est pris en charge par une activité de mise en commun, c'est-à-dire, ce qui est rendu commun par elle. [...] Aucune chose n'est, en soi ou par nature, commune, seules les pratiques collectives décident ultimement du caractère commun d'une chose ${ }^{84}$.

Elinor Ostrom, quant à elle, a mis en lumière qu'un commun est composé de trois éléments pour son fonctionnement ${ }^{85}$. Dans le domaine de l'environnement, ces éléments sont les suivants. Il s'agit d'un objet composite (universalité de fait), indépendant des frontières juridico-politiques, susceptible d'usure et de détérioration alors que vital pour l'homme et pour la pérennité de l'espèce humaine, donc essentiel. Cet objet a une fonction sociale fondamentale. Sur cet objet existe alors une pluralité et/ou diversité de droits d'usage (accès et prélèvement) rivaux et fondamentaux sur l'objet bénéficiant à un/des ensemble(s) de personnes de taille plus ou moins importante (population autochtone, citoyens, environnants). Pour elle la comunauté est une communauté fermée. In fine, une gouvernance de la communalité doit veiller au respect d'un double objectif ; la préservation et la pérennité de la chose afin de ne pas dégrader, détruire ce qui est vital pour l'homme et l'effectivité du droit d'usage et de l'obligation de conservation corrélative nécessaires à la survie de l'homme (individu comme collectif, générations présentes comme futures).

Il est ainsi clair que l'environnement naturel ou la biodiversité ne sont pas des communs mais peuvent être l'objet d'un commun. Nous distinguerons deux catégories de communs naturels, les communs universels et les communs que nous qualifierons de secondaires ou de spatialisés (2).

\footnotetext{
${ }^{84}$ P. Dardot et C. Laval, Commun. Essai sur la révolution au XXI siècle, Paris, La découverte, 2014, p 233 et s

${ }^{85}$ Elinor Ostrom, Gouvernance des biens communs, LLN-Paris, éd. De boeck, 2010
} 
Quelle est l'apport de cette distinction?

Les communs universels doivent représenter la matrice des autres communs naturels, le plus souvent spatialisés. Les principes érigés pour gouverner ce commun universel doivent être perçus comme un régime primaire s'imposant et pouvant influer non seulement sur les régimes secondaires des communs spatialisés mais encore sur les spécimens ou parcelles appropriés.

\section{Les communs naturels universels}

Les communs universels à la façon des beni communi italiens de la commission S. Rodota et tels qu'exposés par B. Coriat à Bruxelles ${ }^{86}$ correspondent aux choses communes de l'article 714 du Code civil, en ce qu'ils « constituent des éléments primordiaux de l'environnement à destination universelle ${ }^{87}$.

Les objets identifiables comme relevant des communs universels sont de manière non exhaustive l'eau libre, l'air, la biodiversité, l'écosystème planétaire.

Ce sont des objets nécessaires à la survie des générations présentes et futures, une atteinte irréversible à ces choses est une atteinte à la condition humaine des générations présentes et futures. Ce sont des entités qui sont hors maitrise de l'État ou des États, qui peuvent paraitre très abstraites, comme la biodiversité ${ }^{88}$ et qui regrouper diverses autres entités. Elles échappent aux limites imposées par les frontières politico-juridiques car elles sont hors propriété publique ou privée absolue et exclusive. Leur marchandisation n'est envisageable ni du point de vue matériel ni du point éthique et de la justice sociale. L'accès doit être ouvert à tous, la communauté concernée n'est pas fermée mais ouverte.

L'usage commun à tous et l'obligation de conservation de la chose commune par les cousagers sont les deux grands principes directeurs du régime juridique analysé plus haut relatif à la chose commune qui ne peut faire l'objet d'exclusivité. Ils doivent être appréhendés comme le régime primaire applicable à ces communs universels. Primaire dans le sens où ces principes pourront influer sur le régime juridique des communs spatialisés, voire sur les spécimens d'espèces, parcelles, portions rattachés à la chose commune et donc aux communs universels.

Dans ce contexte, le droit d'usage est vital pour chacun et commun à tous. L'intérêt en cause est commun car transindividuel mais il est aussi diffus dans le sens où les individus usagers n'ont pas besoin et ne peuvent être identifiés si les générations futures sont concernées.

\footnotetext{
${ }^{86}$ B. Coriat, «Exposer la pluralité de sens : un dictionnaire des communs » in séminaire organisé par D. Misonne sur «Actualités des communs en droit de l'environnement et de la culture, 28 novembre 2017, CEDRE, Université Saint Louis, Bruxelles, V. également Benjamin Coriat, Le retour des communs, Paris, éd. LLL les liens qui libèrent 2015

${ }^{87}$ J.-C. Saint-Pau, «Le patrimonial et l'extrapatrimonial à l'épreuve des questions environnementales », Les notions fondamentales de droit privé à l'épreuve des questions environnementales, (dir. M. Mekki), Bruxelles, éd. Bruylant, Droit (s) et développement durable, 2016, p.104

${ }^{88}$ Article L110-1 I. al. 3 « On entend par biodiversité, ou diversité biologique, la variabilité des organismes vivants de toute origine, y compris les écosystèmes terrestres, marins et autres écosystèmes aquatiques, ainsi que les complexes écologiques dont ils font partie. Elle comprend la diversité au sein des espèces et entre espèces, la diversité des écosystèmes ainsi que les interactions entre les organismes vivants »
} 
Il n'exclut pas la propriété de parcelles ou de composants de cet objet mais oblige en revanche ce propriétaire à tenir compte de ce que ces parcelles/composantes font partie d'un tout universel (chose commune), ce qui peut réduire son propre droit sur l'objet de propriété. Ainsi, l'usage commun prime sur le droit de propriété exclusif ${ }^{89}$ qui ne joue qu'un rôle très subsidiaire. En effet ce droit de propriété exclusif ne peut porter que sur les fruits de la chose commune (propriétaire d'un spécimen ou d'une parcelle).

En termes de gouvernance, ces communs universels posent question puisqu'il n'existe pas d'entité juridique mondiale représentant ces intérêts ou ceux des co-usagers. Cependant, la France en particulier peut faire respecter l'environnement à travers la Charte, les différentes législations environnementales et par le biais de l'application de l'article 714 du Code civil dans sa lecture actualisée. L'État doit permettre une application de ce régime primaire (usage commun, conservation par tous) de l'objet des communs universels. De même, en raison de ce régime primaire dégagé et qui dépasse les limites du territoire français, le juge à différents échelons extranationaux comme locaux peut d'ores et déjà gérer certains conflits. Nous avons analysé sommairement et à notre mesure dans quel cadre civil français ces actions populaires ou en défense d'intérêt collectif ou diffus peuvent se développer. En effet, l'obligation de conservation pour l'usager correspondant à un droit à la conservation pour ses co-usagers et le risque d'irréversibilité de l'atteinte doivent nécessairement permettre un recours en justice en défense d'un intérêt commun ${ }^{90}$. Ces droits et obligations peuvent entraîner la responsabilité des usagers, du propriétaire ou du gardien, dans ce cas usagers privilégiés, voire de l'État au regard de son action possible pour préserver ces choses communes envers les co-usagers. L'accès au juge devrait se faire grâce à la notion d'intérêt collectif environnemental, ou intérêt commun ou intérêt diffus.

Et le juge devra imposer le respect de la chose commune mais également cet usage commun vital et donc l'impossible exclusion d'individus du bénéfice de ces communs universels.

Selon B. Coriat, l'institutionnalisation de ces communs universels doit permettre la garantie de la préservation et de l'accès. Pour les institutionnaliser, et donc en préserver l'existence et l'usage ${ }^{91}$, il faudrait, selon lui, adopter une déclaration des communs universels sur le modèle de la Déclaration des Droits de l'Homme et du Citoyen ${ }^{92}$.

Ces communs doivent être distingués d'autre modèle de communs existants. En effet, à la différence de ce communs universels, il existe des communs spatialisés tels que décrits par

\footnotetext{
${ }^{89}$ Selon P. Charbonnier et D. Festa, « dès lors qu'on reconnait la dimension collective et même sociale du rapport à certains choses (ici la chose commune) alors la mission du droit consiste à reconnaitre et à protéger ce caractère collectif contre les formes d'appropriation exclusive » in «Biens communs, beni comuni », préc.

${ }^{90}$ C. Cournil et A-S Tabau, « Nouvelles perspectives pour la justice climatique », RJE 4/2015, p. 675, à propos de l'affaire Urgenda en matière de pollution de l'air.

${ }^{91}$ Voir dans le même sens les initiatives visant à la reconnaissance des droits humains, notamment na déclaration universelle des droits humains dont l'article VIII déclare que «l'humanité a droit à la préservation des biens communs en particulier l'eau, l'air et le sol et à l'accès universel et effectif aux ressources vitales. Les générations futures ont droit à leur transmission », La déclaration universelle des droits humains éd. Duchène 2016.

${ }^{92}$ B. Coriat, «Exposer la pluralité de sens : un dictionnaire des communs » in séminaire organisé par D. Misonne sur « Actualités des communs en droit de l'environnement et de la culture, 28 novembre 2017, CEDRE, Université Saint Louis, Bruxelles
} 
Elinor Ostrom ${ }^{93}$ ou pouvant faire l'objet d'une transpropriation selon l'expression de François $\mathrm{Ost}^{94}$.

\section{Les Communs spatialisés}

Un fleuve, une forêt, un écosystème local, une population localisée de spécimens d'une espèce peuvent être des objets constitutifs de ces communs spatialisés ou secondaires. Ils trouvent nécessairement leur origine et leur capacité de fonctionnement dans les choses communes matricielles, objets des communs universels. Chacune de ces entités naturelles fait en effet l'objet d'un faisceau d'usages variés : loisir, travail, besoin physique ou spirituel. Le groupe d'humains dépendant de cette entité naturelle est très variable mais il peut être globalement cerné, structuré et organisé (une population autochtone, les pêcheurs d'une pêcherie). L'intérêt en cause est commun, car trans-individuel, mais concerne un groupe précis. Parmi les titulaires de ces usages peuvent se trouver un ou des propriétaires. L'entité naturelle étant spatialisée est forcément sous juridiction nationale et peut faire l'objet d'un droit de propriété public ou privé. Dans ce cas, droit de propriété et droits d'usage peuvent s'exercer simultanément sur cet objet ou se superposer ${ }^{95}$. Le propriétaire peut avoir plus de droit que les autres usagers mais il a une obligation renforcée de conservation et d'affectation ${ }^{96}$ (propriété à charge) et doit laisser l'accès aux autres usagers (propriété inclusive). Il est alors le dépositaire de la chose commune. Sylvie Paquerot relève cependant que dans ce cadre qui est celui exploré par E. Ostrom, le commun n'est pas universel, de sorte qu'une communauté peut avoir le privilège de l'accès à un territoire à l'exclusion d'une autre ${ }^{97}$.

Une gouvernance peut être organisée par le groupe identifié en lien avec le ou les États souverains en fonction des besoins des usagers sous différents régimes, tels les patrimoines mondiaux de l'UNESCO, les monuments historiques mais également des conventions entre l'État et une collectivité de citoyens, ...

Cependant ces communs spatialisés doivent s'articuler avec la catégorie de communs universels regroupant les choses communes ayant un régime juridique qui doit s'imposer. $\mathrm{Ce}$ régime de la chose commune peut de ce fait être qualifié de primaire dans la mesure où cet usage commun et l'obligation de conservation corrélative doivent être respectés quels que soient les régimes spécifiques, secondaires des communs spatialisés et peut influer jusqu'au régime applicable aux éléments détachables (colonie d'oiseaux). En effet, par exemple

\footnotetext{
${ }^{93}$ Elinor Ostrom, Gouvernance des biens communs, LLN-Paris, éd. De boeck, 2010

${ }^{94} \mathrm{~F}$. Ost, « Responsabilité, après nous le déluge? » in La nature hors la loi, l'écologie à l'épreuve du droit, Paris, éd. La découverte, 1995, p. 323.

${ }^{95}$ Selon, F. Ost, «le propriétaire conserve la plupart de ses prérogatives, mais certaines d'entre elles se trouvent désormais subordonnées à l'objectif de conservation. Comme si son bien était géré de l'extérieur, le "propriétaire ne dispose plus nécessairement de la même intensité d'emprise sur chacun des aspects de son bien ». F. Ost, « Responsabilité, après nous le déluge? » in La nature hors la loi, l'écologie à l'épreuve du droit, Paris, éd. La découverte, 1995 , p. 323

96 A. Chaigneau, «Une propriété affectée au commun», in (dir A. Chaigneau) Fonctions de la propriété et commun, regards comparatistes, éd. Soc de Leg. Comparée 2017, Paris, LGDJ, p. 65

${ }^{97}$ S. Paquerot, «Commun et bien commun », in (dir A. Chaigneau) Fonctions de la propriété et commun, regards comparatistes, éd. Soc de Leg. Comparée 2017, Paris, LGDJ, p.21
} 
l'appartenance de la biodiversité à la catégorie de communs universels a des incidences sur un écosystème forestier pouvant faire l'objet d'une communalité spatialisée (forêt de Soignes à Bruxelles) puisque le droit d'usage et l'obligation de conservation sur la biodiversité sont fondamentaux.

Cette théorie des communs peut paraitre théorique ou utopique, elle fait cependant partie d'un courant qui tente d'apporter des réponses à l'état toujours plus dégradé de notre planète, à la réduction des ressources naturelles et à l'effondrement de biodiversité qui ne peut entrainer que tension et barbarie ${ }^{98}$ si rien ne change ${ }^{99}$. Dans ces deux catégories de communs, la propriété n'est pas exclue, elle est cependant nécessairement réduite en fonction de la nécessité de tous de bénéficier des utilités environnementales, réduite quant aux objets sur lesquels elle peut porter, et quant à son ampleur (exclusive sur les fruits, à charge et non exclusive ou inclusive sur les objets de communs spatialisés). L'État n'est pas non plus exclu, il peut même contribuer à la bonne gouvernance de ces communs, même s'il n'est pas forcément l'interlocuteur principal ou majeur, ne serait-ce qu'en favorisant une application effective et adaptée de l'article $714 \mathrm{du}$ Code civil français ${ }^{100}$. Il faut, en revanche, que l'État accepte une vision plus participative des citoyens, qu'il leur laisse éventuellement la part belle, ce que n'est apparemment pas prêt à faire l'État français au regard des évènements récents sur le site de l'ancien projet d'aéroport de Notre Dame Des Landes.

Marie-Pierre Camproux Duffrène

Professeure de droit privé,

Laboratoire SAGE UMR 7363, Université de Strasbourg-CNRS

\footnotetext{
${ }^{98}$ Serge Gutwirth et Isabelle Stengers, « le droit à l'épreuve de la résurgence des commons, RJE 1/2016, p. 343

${ }^{99}$ V. E. Lesourt Survivre à l'anthropocène, Paris, PUF, L'écologique en questions, 2018.

${ }^{100}$ Cet article apparaît d'ailleurs aujourd'hui bien isolé dans le Code civil et n'est pas à l'abri d'une réforme le supprimant alors qu'il mériterait au contraire d'être précisé et ses conséquences légalisées.
} 